${ }^{5}$ ITQB-NOVA, Instituto de Tecnologia Química e Biológica António Xavier, Oeiras, Portugal

Background: Early diagnosis of axial Spondyloarthritis (axSpA) represents a major clinical challenge nowadays. Increasing evidence has determined that early diagnosis, prompt treatment initiation and early achievement of remission are the best predictors of long-term clinical, functional and radiographic outcomes. New tools to support the diagnosis are needed.

Objectives: This study aims to identify differentially expressed genes that may improve the current clinical diagnosis approach for early axSpA.

Methods: A cross-sectional study was conducted on 50 participants, 25 patients with axSpA (according to ASAS criteria) and 25 Healthy Controls, matched by gender, age and levels of physical activity. Peripheral blood samples were collected and RNA-Seq technology was performed. Normalization of raw data, and identification of differentially expressed genes was obtained using edgeR and limma-voom R packages. Gene Set Enrichment Analysis (GSEA) and Functional Enrichment analysis using Gene ontology (GO) and Kyoto Encyclopedia of Genes and Genomes (KEGG) annotations were also performed. A number of Differently Expressed Genes were highlighted.

Results: 311 genes were identified as being significantly differentially expressed between patients and controls. In details, 129 downregulated (7 genes have fold change more than 1) and 182 upregulated genes ( 3 genes have fold change more than 1) are highlighted. These genes are mostly involved in Myogenesis, Innate Immune Signalling and JAK/STAT pathways. Several genes with functions of skeletal muscle development and muscle contraction were identified.

Conclusion: The evidence disclosed that regulation of muscle development and contraction may be also engaged in physiopathology mechanisms of axSpA. These new cues open new perspectives for diagnosis and therapeutic approaches in axSpA.

Acknowledgments: To all patients and healthy people who participate in MyoSpA study

Disclosure of Interests: Atlas Mashayekhi Sardoo: None declared, Daniel Sobral: None declared, Lucia Domingues: None declared, Santiago Rodrigues-Manica Speakers bureau: Jansse, MSD, Novartis, Rita Pinheiro Torres: None declared, Agna Neto: None declared, Patricia Alves: None declared, Julia Costa: None declared, Ana Rita Grosso: None declared, Jaime Branco Speakers bureau: Vitoria, Fernando Pimentel dos Santos Speakers bureau: Novartis, Pfizer, Biogen, Vitoria,

DOI: 10.1136/annrheumdis-2020-eular.5331

\section{THU0022 DIFFERENTIAL DNA METHYLATION AS A PREDICTOR OF TOCILIZUMAB RESPONSE IN RHEUMATOID ARTHRITIS PATIENTS}

N. Nair ${ }^{1}$, D. Plant ${ }^{1,2}$, J. Isaacs ${ }^{3}$, A. Morgan ${ }^{4}$, K. Hyrich ${ }^{1}$, A. Barton ${ }^{1,2}$, A G. Wilson ${ }^{5}$ on behalf of MATURA. ${ }^{1}$ The University of Manchester, Versus Arthritis Centre for Genetics and Genomics and Centre for Epidemiology, Manchester, United Kingdom; ${ }^{2}$ NIHR Manchester Biomedical Research Unit, Manchester, United Kingdom; ${ }^{3}$ Newcastle University, NIHR Newcastle BRC, Newcastle upon Tyne Hospitals NHS Foundation Trust, Newcastle, United Kingdom: ${ }^{4}$ Leeds School of Medicine, University of Leeds and NIHR Leeds BRC, Leeds, United Kingdom; ${ }^{5}$ University College Dublin, School of Medicine and Medical Science, Dublin, Ireland

Background: Tocilizumab (TCZ) is a biological disease-modifying antirheumatic drug that blocks IL-6 signalling and is effective in ameliorating disease activity in rheumatoid arthritis (RA). However, approximately $50 \%$ of patients do not respond adequately to TCZ and some patients report adverse events. Considering there is growing evidence that DNA methylation is implicated in RA susceptibility and response to some biologics $(1,2)$, we investigated DNA methylation as a candidate biomarker for response to TCZ in RA.

Objectives: To identify differential DNA methylation signatures in whole blood associated with TCZ response in patients with RA.

Methods: Epigenome-wide DNA methylation patterns were measured using the Infinium EPIC BeadChip (Illumina) in whole blood-derived DNA samples from patients with RA. DNA was extracted from blood samples taken pre-treatment and following 3 months on therapy, and response was determined at 6 months using the Clinical Disease Activity Index (CDAI). Patients who had good response $(n=10)$ or poor response $(n=10)$ to TCZ by 6 months were selected. Samples from secondary poor responders $(n=10)$ (patients who had an improvement of CDAl and were in remission at 3 months, followed by a worsening of CDAl at 6 months) were also analysed. Differentially methylated positions and regions (DMPs/DMRs) were identified using linear regression, adjusting for gender, age, cell composition, smoking status, and glucocorticoid use. Gene Set Enrichment Analysis (GSEA) was used to identify significant pathways associated with response and Functional Epigenetic Module analysis of interactome hotspots in regions of differential methylation.

Results: 20 DMPs were significantly associated with response status at 6 months in the pre-treatment samples. Another 21 DMPs were associated with response in the 3 month samples. Within good responders, 10 DMPs showed significant change in methylation level between pre-treatment and the 3 month samples (unadjusted P-value $<10^{-6}$ ). One DMP, cg03121467, was significantly less methylated in good responders compared to poor responders in the pre-treatment samples. This DMP is close to EPB41L4A and thought to have a role in $\beta$-catenin signalling. GSEA of DMRs in non- and secondary non- responders identified histone acetyltransferase pathways and included the KAT2A gene, which is a repressor of NF-kB. Additional analysis of interaction hotspots of differential methylation identified significant interactions with STAMBP and PTPN12 associated with response status.

Conclusion: These preliminary results provide evidence that DNA methylation patterns may predict response to TCZ. Validation of these findings in other larger data sets is required.

\section{References:}

[1] Liu,Y., Aryee,M.J., Padyukov,L., Fallin,M.D., Hesselberg,E., Runarsson,A. Reinius,L., Acevedo,N., Taub,M., Ronninger,M., et al. (2013) Epigenome-wide association data implicate DNA methylation as an intermediary of genetic risk in rheumatoid arthritis. Nat. Biotechnol., 31, 142-147.

[2] Plant,D., Webster,A., Nair,N., Oliver,J., Smith,S.L., Eyre,S., Hyrich,K.L., Wilson,A.G., Morgan,A.W., Isaacs,J.D., et al. (2016) Differential Methylation as a Biomarker of Response to Etanercept in Patients With Rheumatoid Arthritis. Arthritis Rheumatol. (Hoboken, N.J.), 68, 1353-60.

Disclosure of Interests: Nisha Nair: None declared, Darren Plant: None declared, John Isaacs Consultant of: AbbVie, Bristol-Myers Squibb, Eli Lilly, Gilead, Janssen, Merck, Pfizer, Roche, Ann Morgan Grant/research support from: I have received a grant from Roche Products Ltd to establish a registry for GCA patients treated with tocilizumab., Consultant of: I have undertaken consultancy work for Roche, Chugai, Regeneron, Sanofi and GSK in the area of GCA therapeutics., Speakers bureau: I have presented on tocilizumab therapy for GCA and glucocorticoid toxicity on behalf of Roche products Itd. Kimme Hyrich Grant/research support from: Pfizer, UCB, BMS, Speakers bureau: Abbvie, Anne Barton Consultant of: AbbVie, Anthony G Wilson: None declared

DOI: 10.1136/annrheumdis-2020-eular.4394

\section{THU0023 \\ DETAILED PROFILE OF CO-OCCURRENCE OF RELAPSING POLYCHONDRITIS AND AUTOIMMUNE THYROID DISEASE}

T. Nakajima ${ }^{1}$, H. Yoshifuji ${ }^{2}$, Y. Yamano ${ }^{3}$, H. Handa ${ }^{4}$, K. Ohmura ${ }^{2}$, T. Mimori ${ }^{2}$, C. Terao ${ }^{5,6,7} .{ }^{1}$ Tazuke Kofukai Medical Research Institute, Kitano Hospital, Department of Clinical Immunology and Rheumatology, Osaka, Japan; ${ }^{2}$ Kyoto University Graduate School of Medicine, Department of Rheumatology and Clinical Immunology, Kyoto, Japan; ${ }^{3}$ St. Marianna University School of Medicine, Department of Rare Diseases Research, Institute of Medical Science, Kawasaki, Japan; ${ }^{4}$ St. Marianna University School of Medicine, Division of Respiratory and Infectious Diseases, Department of Internal Medicine, Kawasaki, Japan; ${ }^{5}$ RIKEN Center for Integrative Medical Sciences, Laboratory for Statistical and Translational Genetics, Yokohama, Japan; ${ }^{6}$ Shizuoka General Hospital, Clinical Research Center, Shizuoka, Japan; ${ }^{7}$ The School of Pharmaceutical Sciences, University of Shizuoka, The Department of Applied Genetics, Shizuoka, Japan

Background: Relapsing polychondritis (RP) is a rare inflammatory disease which is characterized by recurrent inflammation and destruction of cartilage tissues. RP also has the profile of autoimmune disease and is often complicated with other autoimmune disease. Autoimmune thyroid disease (AITD) is one of common autoimmune diseases, which consists of Graves' disease (GD) and Hashimoto's thyroiditis (HT). While RP is reported to be complicated with AITD ${ }^{1)}$, there has been no study on detailed profile of co-occurrence of RP and AITD.

Objectives: We aimed to reveal whether there is common (statistically significant) co-occurrence of RP and AITD. We also analyzed clinical and genetic profiles characterizing the co-occurrence.

Methods: We recruited 117 patients with RP and checked their medical records in order to obtain the information about compilation of AITD and clinical features. In addition, we genotyped Human Leucocyte Antigen (HLA) A, B Cw, DRB1, DQB1 and DPB1 alleles for 88 of the 117 patients. Co-occurrence ratio was compared with prevalence of AITD in the Japanese population. Associations of co-occurrence of AITD with clinical manifestations or HLA alleles were analyzed among the patients. 
Results: Among the 117 patients with RP, 5 (4.3\%) and 6 (5.1\%) patients had GD and HT, respectively. Patients with RP were more likely to be complicated with GD $\left(p=1.04 \times 10^{-3}\right.$, OR: $\left.7.15,95 \% \mathrm{Cl} 2.68 \sim 18.14\right)$ but not with HT $(p=0.50$, $95 \% \mathrm{Cl} 0.59 \sim 1.27$ ), compared with prevalence in general Japanese population $\left(0.62 \%\right.$ and $5.9 \%$, respectively $\left.{ }^{2}\right)$. RP patients with GD showed a trend to have nasal involvement ( $100 \%$ vs $45.5 \%, \mathrm{p}=0.023$, OR: $2.58,95 \% \mathrm{Cl} 1.09 \sim \infty)$. We did not observe any differences in clinical manifestation in patients with RP and HT. HLA- DPB $1{ }^{*} 02: 02$ demonstrated a trend toward GD complication ( $20 \%$ vs $2.3 \%$, $\mathrm{p}=0.035$, OR: $10.41,95 \% \mathrm{Cl} 1.23 \sim 65.38)$. There were no association of HLA in the complication of HT among patients with RP.

Conclusion: Patients with RP have high co-occurrence ratio of GD. Patients with the two diseases may be characterized by nasal involvement and HLA-DPB $1{ }^{*} 02: 02$

References:

[1] Kung AW et al. Graves' ophthalmopathy and relapsing polychondritis. Clin Exp Rheumatol. 1995 Jul-Aug;13(4):501-3.

[2] Nagataki $S$ et al. Thyroid diseases among atomic bomb survivors in Nagasaki. JAMA. 1994 Aug 3;272(5):364-70.

Disclosure of Interests: Toshiki Nakajima Speakers bureau: Bristol-Myers Squibb and Novartis, Hajime Yoshifuji Grant/research support from: Astellas Pharma. (Outside the field of the present study.), Speakers bureau: Chugai Pharmaceutical. (Outside the field of the present study.), Yoshihisa Yamano: None declared, Hiroshi Handa: None declared, Koichiro Ohmura Grant/ research support from: Astellas Pharma, AYUMI Pharmaceutical, Chugai Pharmaceutical, Daiichi Sankyo, Eisai, Japan Blood Products Organization, Mitsubishi Tanabe Pharma, Nippon Kayaku, Nippon Shinyaku, Sanofi, and Takeda Pharmaceutical., Speakers bureau: AbbVie, Actelion Pharmaceuticals Japan, Asahi Kasei Pharma, AYUMI Pharmaceutical, Bristol-Myers Squibb, Chugai Pharmaceutical, Eisai, Eli Lilly and Company, GlaxoSmithKline, Janssen Pharmaceutical, Mitsubishi Tanabe Pharma, Novartis Pharma, and Sanofi., Tsuneyo Mimori: None declared, Chikashi Terao Grant/research support from: Actelion, Speakers bureau: Asteras, Asahi Kasei Pharma, Ono and Tanabe-Mitsubishi

DOI: 10.1136/annrheumdis-2020-eular.5349

\section{THU0024 METHYLATION ANALYSIS OF VITAMIN D SIGNALING PATHWAY GENES IN RHEUMATOID ARTHRITIS PATIENTS}

E. Punceviciene ${ }^{1,2,3}$, J. Gaizevska ${ }^{4,5}$, R. Sabaliauskaite ${ }^{5}$, L. Venceviciene ${ }^{6,7}$, D. Vitkus ${ }^{8,9}$, S. Jarmalaite ${ }^{4,5}$, I. Butrimiene ${ }^{1,2,3}$. ${ }^{1}$ Centre of Rheumatology, Vilnius University Hospital Santaros Klinikos, Vilnius, Lithuania; ${ }^{2}$ State Research Institute Centre for Innovative Medicine, Vilnius, Lithuania; ${ }^{3}$ Clinic of Rheumatology, Traumatology Orthopaedics and Reconstructive Surgery, Institute of Clinical Medicine of the Faculty of Medicine, Vilnius University, Vilnius, Lithuania; ${ }^{4}$ Institute of Biosciences, Life Sciences Center, Vilnius University, Vilnius, Lithuania; ${ }^{5}$ Laboratory of Genetic Diagnostics, National Cancer Institute, Vilnius, Lithuania; ${ }^{6}$ Clinic of Internal Medicine, Family Medicine and Oncology, Faculty of Medicine, Vilnius University, Vilnius, Lithuania; ${ }^{7}$ Centre of Family Medicine, Vilnius University Hospital Santaros Klinikos, Vilnius, Lithuania; ${ }^{8}$ Institute of Biomedical Sciences of the Faculty of Medicine, Vilnius University, Vilnius, Lithuania; ${ }^{9}$ Centre of Laboratory Medicine, Vilnius University Hospital Santaros Klinikos, Vilnius, Lithuania

Background: Vitamin D is known for its immunomodulatory and epigenome interacting effects. Vitamin $D$ deficiency is frequently observed in rheumatoid arthritis (RA) patients compared to healthy controls, is also named as a potential risk factor in RA ethiopatogenesis and may alter DNA methylation of certain genes [1,2]. Still, causality of vitamin $D$ deficiency in RA patients needs to be elucidated.

Objectives: The aim of the study was to evaluate relationship between DNA methylation status of vitamin D related genes (VDR, CYP24A1, CYP2R1), miRNA-155 expression, vitamin D level and its association with RA.

Methods: $\mathrm{CpG}$ islands in promoter region of the VDR, CYP24A1, CYP2R1 genes were chosen for DNA methylation analysis by means of pyrosequencing. DNA from blood mononuclear cells of 31 RA patients and 31 age and sex matched healthy controls was assessed for methylation pattern after informed consent was obtained in Vilnius university Hospital Santaros klinikos Centre of Rheumatology. For miRNA analysis quantitative reverse transcription PCR was used. Chemiluminescent microplate immunoassay was used to asses $25(\mathrm{OH})$ D serum levels.

Results: $25(\mathrm{OH}) \mathrm{D}$ concentrations varied from deficiency $(<50 \mathrm{nmol} / \mathrm{l})$, insufficiency $(50-75 \mathrm{nmol} / \mathrm{l})$ to normal range $(\geq 75-100 \mathrm{nmol} / \mathrm{l})$ in RA (mean $47.49 \mathrm{nmo}$ $\mathrm{l} / \mathrm{l} ; \mathrm{SD} \pm 27.93$ ) and healthy controls (mean $57.38 \mathrm{nmol} / \mathrm{l} ; \mathrm{SD} \pm 29.93)$ ). CYP24A1 methylation level was significantly higher in comparison to VDR $(\mathrm{p}<0.0001)$ and CYP2R1 $(p<0.0001)$ genes in both groups. CYP24A1 hypermethylation was also observed in older subjects $(p=0.012)$. The study demonstrated a significant positive correlation between vitamin $\mathrm{D}$ concentration and VDR, CYP2R1 genes methylation intensity $\left(r^{2}=0.31, p=0.014 ; r^{2}=0.25, p=0.042\right.$, respectively) However, gene methylation frequency and methylation intensity showed no significant difference between RA patients and healthy controls (VDR - 2.4 vs 2.6 \%, CYP24A1 - 16.6 vs $15.3 \%$, CYP2R1 - 2.6 vs $2.6 \%$ ( $>0.05)$. To note, miRNA-155 expression negatively correlated with CYP24A1 methylation inten sity $\left(r^{2}=-0.43, p=0.009\right)$.

Conclusion: Our study identified significant associations between the VDR and CYP2R1 promoter methylation and vitamin D concentration. However no significant differences in DNA methylation pattern between RA patients and healthy controls were detected. MiR-155 expression was associated with CYP24A1 methylation level, confirming its possible involvement in vitamin D metabolism. The data of our study suggests that epigenetic phenomena are significantly involved in vitamin D metabolism and may have an indirect effect on RA ethiopatogenesis.

References:

[1] Jeffery LE, et al. Nat Rev Rheumatol. 2016,12.4:201.

[2] Fetahu IS et al. Front Physiol. 2014,5:164

Acknowledgments: This project has received funding from the Research Council of Lithuania (LMTLT), agreement No. S-MIP-17-12.

Disclosure of Interests: None declared

DOI: 10.1136/annrheumdis-2020-eular.2593

\section{THU0025 MICRO-RNA DIFFERENTIALLY REGULATE THE ALTERNATIVE PRTN3-MRNA IN GRANULOMATOSIS WITH POLYANGIITIS}

N. Reichard ${ }^{1}$, A. Kerstein-Staehle ${ }^{1}$, A. Müller ${ }^{1}$, G. Riemekasten ${ }^{1}$, P. Lamprecht ${ }^{1}$, S. Schinke $1 .{ }^{1}$ University Lübeck, Rheumatology and Clinical Immunology, Lübeck, Germany

Background: Micro-RNAs (miRNA) are short non-coding RNAs that regulate inflammation mostly by translational repression. Previously, we screened 847 miRNAs in nasal tissue from GPA patients and found a disease associated alteration of miRNA expression compared to healthy controls and chronic rhinosinusitis. MiR-184 was most over expressed in nasal tissue from GPA (13.4x). The dual-luciferase reporter assay confirmed a significant reduction of Proteinase-3 (PRTN3) expression by miR-184 (1).

PRTN3 transcripts with an alternative 3' untranslated region (UTR) have been described in GPA (2). The pathophysiological relevance of this alternative transcript remains unclarified.

Objectives: To identify new miRNA targets of potential pathophysiological relevance in GPA, we validated the effect of the 21 most dysregulated miRNAs on the mRNA of PRTN3. Further, we included the alternative PRTN3 mRNA in our screen to look for new regulatory differences.

Methods: The inhibitory capacity of miRNAs on Proteinase-3 mRNA was estimated by a dual-luciferase reporter system. The sequences of the alternative (132bp longer) and the regular 3'UTR-PRTN3 were cloned and inserted into the pmirGLO vector and co-transfected with 21 miRNA mimics into HeLa cells. Co-transfection with Caenorhabditis elegans miRNA 67 mimic (cel-miR-67) was used as negative control. Statistical significance was evaluated by students t-test adjusted for multiple comparisons (Holm-Sidak).

Results: For 18 of 21 investigated miRNAs no effects could be observed on the alternative and the regular 3'UTR-PRTN3. But there were remarkable differential effects of let-7f, miR-184 and miR-708. Let-7f (-29,2\%) and miR-708 (-23,6\%) both showed a suppression of the alternative 3'UTR-PRTN3 but no effect on the regular 3'UTR-PRTN3 while miR-184 only suppressed the regular 3'UTR (-17,5 $\%)$ and not the alternative variant (fig. 1-2).

Conclusion: Disease specific miRNA signatures together with an increased PRTN3 level and in alternative PRTN3 mRNA in GPA suggest a dysregulation of PRTN3 expression in GPA. To our knowledge this is the first analysis in GPA showing that miRNAs can differentially regulate the expected and the alternative 3'UTR variants of PRTN3-mRNA. As miR-184 is markedly upregulated in GPA, a repression of PRTN3 is to be anticipated, possibly as a reaction to previous neutrophil activation with PRTN3 overexpression. Our findings also strengthen the potential pathophysiological role of the alternative PRTN3 mRNA.

\section{References:}

[1] Schinke $S$ et al PROTEINASE-3 REGULATING MICRO-RNA IN GRANULOMATOSIS WITH POLYANGIITIS. Ann Rheum Dis 2019 (78 Suppl 2):437 